\title{
Well-width dependence of the exciton-phonon scattering in thin InGaAs/GaAs single quantum wells
}

Borri, Paola; Langbein, Wolfgang Werner; Hvam, Jørn Märcher; Martelli, F.

Published in:

Summaries of papers presented at the International Quantum Electronics Conference

Link to article, DOI:

10.1109/IQEC.1998.680216

Publication date:

1998

Document Version

Publisher's PDF, also known as Version of record

Link back to DTU Orbit

Citation (APA):

Borri, P., Langbein, W. W., Hvam, J. M., \& Martelli, F. (1998). Well-width dependence of the exciton-phonon scattering in thin InGaAs/GaAs single quantum wells. In Summaries of papers presented at the International Quantum Electronics Conference (pp. 103-104). IEEE. https://doi.org/10.1109/IQEC.1998.680216

\section{General rights}

Copyright and moral rights for the publications made accessible in the public portal are retained by the authors and/or other copyright owners and it is a condition of accessing publications that users recognise and abide by the legal requirements associated with these rights.

- Users may download and print one copy of any publication from the public portal for the purpose of private study or research.

- You may not further distribute the material or use it for any profit-making activity or commercial gain

- You may freely distribute the URL identifying the publication in the public portal 


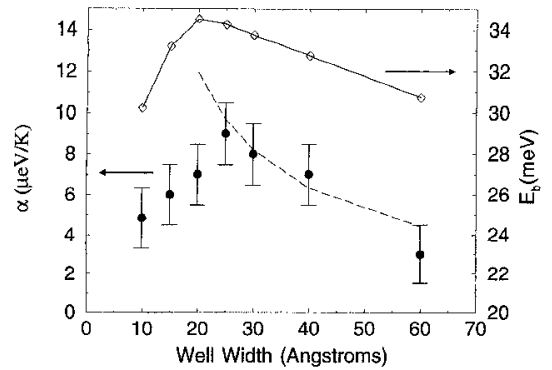

QWE2 Fig. 1. Well-width dependence of the exciton-acoustic-phonon scattering parameter $\alpha$ (observed, solid points; modeled, dashed line) and well-width dependence of the exciton binding energy (solid line).

lium arsenide (GaAs) substrates. All samples have a constant cadmium fraction of $18 \%$ and $80 \AA$ barrier width. The samples have 20 wells of nominal widths: $10,15,20,25,30,40$, and 60 $\AA$. Exciton-resonant, femtosecond TI-FWM was carried out on each sample at five temperatures between 4 and $70 \mathrm{~K}$ and five excitation densities between 0.5 and $2.5 \times 10^{9} \mathrm{~cm}^{-2}$. Acquisition of this large data set of 175 TIFWM delay scans is made possible by the use of fastscan time-delay techniques. Exponential fits to the data, assuming a photon-echo configuration, yield measured values of $\gamma$. Linear fits to these $\gamma$ values at low temperature $(<50$ K) for a given excitation density provide the exciton-acoustic-phonon scattering parameter, $\alpha$, for a particular sample and confirmed $\alpha$ to be independent of excitation density.

The well-width dependence of the excitonacoustic-phonon scattering parameter, $\alpha$, is shown in Fig. 1. It exhibits a pronounced peak at around $20 \AA$ with lower values on either side. The dashed line in Fig. 1 is obtained from a model based on an infinite depth quantum well ${ }^{3}$ using literature values for the material parameters. Two contributions are included, namely, deformation potential and piezoelectric scattering. The dominant deformation potential scattering rate is inversely proportional to well width and this model accurately reproduces the observed wide-well behavior. The dependence of the scattering rate on well width reflects the spatial extent of the wavefunction, however, in the narrow well regime this extent is not defined simply by the well-width and the infinite-depth quantum-well model breaks down. Qualitatively the inverse well-width dependence should be replaced by an inverse dependence on the spread of the exciton wave function. An accessible measure of this spread is the exciton binding energy. We show in Fig. 1 the exciton-binding energy as determined from low-temperature linear absorption spectra fitted to an eight-band $k . p$ model. The rollover in the exciton binding energy, which is due to the reduced quantum confinement, also peaks at a well width of around $20 \AA$. Thus the reduced exciton-acoustic-phonon scattering in the narrow well regime is due to a reduced quantum confinement.

In conclusion, we have observed that the maximum of both the exciton-acoustic-phonon scattering parameter and the exciton binding energy occur at a similar well width indicating that the exciton-acoustic-phonon scattering rate is also directly correlated to the degree of quantum confinement.

1. J. Shah, Ultrafast Spectroscopy in Semiconductors, (Springer, Berlin 1996).

2. B.K. Ridley, in Hot Carriers in Semiconductor Nanostructures: Physics and Applications, J. Shah, ed. (Academic Press, London 1992).

3. J. Lee, E.S. Kotales, M.O. Vassel, Phys. Rev. B 33, 5512 (I986).

\section{QWE3}

8:30 am

Well-width dependence of the excitonphonon scattering in thin InGaAs/GaAs single quantum wells

P. Borri, W. Langbein, J.M. Hvam,

F. Martelli ${ }^{\star}$, Mikroelektronik Centret, The

Technical University of Denmark, Building 345

East, DK-2800 Lyngby, Denmark; E-mail: paola@mic.dtu.dk

The effect of phonon scattering on both exciton linewidth and dephasing time in bulk semiconductors and confined systems has attracted a lot of interest in the last 15 years. Most of the work in low-dimensional structures has been dedicated to GaAs/AlGaAs quantum well structures; linewidth broadening coefficients of $\sim 2 \mu \mathrm{eV} / \mathrm{K}$ for acoustic phonons and $\sim 10$ $\mathrm{meV}$ for optical phonons are reported by Gammon et al. ${ }^{1}$ Moreover, contradictory results for the well-width dependence of these coefficients are reported.

In this work, we studied the temperature dependence of the exciton dephasing time in three $\mathrm{In}_{0.18} \mathrm{Ga}_{0.82} \mathrm{As} / \mathrm{GaAs}$ single quantum wells, with well thickness $L_{w}$ of $1,1.5$, and 2 $\mathrm{nm}$, by degenerate time-integrated four-wavemixing (TI-FWM) using $100-\mathrm{fs}$ pulses in reflection geometry. The TI-FWM correlation traces clearly show an inhomogeneous broadening in all the samples at low temperature (5 K). In Fig. la we show TI-FWM traces at the heavy-hole exciton transition in the $1.5-\mathrm{nm}$ wide well, for resonant excitation at different temperatures. Around $95 \mathrm{~K}$, the trace shows a decay that is no longer exponential for long delay time, while an exponential trend is recovered for short positive delay at higher temperature. This indicates the transition from inhomogeneous to homogeneous broadening with increasing the temperature, ${ }^{2}$ according to exciton linewidth analysis. The dephasing rate is deduced consequently by dividing the FWM decay rate by a factor of 4 for $T \leq 80 \mathrm{~K}$, and 2 for $\mathrm{T}=110 \mathrm{~K}$, while the transition point is skipped. Also, we measure the density dependence of the dephasing and extrapolate the rate to zero density. The resulting $1 / \mathrm{T}_{2}$ versus temperature is shown in Fig. 1b. The acoustic and optical coefficient of the corresponding linewidth broadening have been deduced by fitting the data, as indicated.

In Fig. $2 \mathrm{a}$ we report the $a$ (acoustic) and $b$ (optical) phonon coefficient versus $\mathrm{L}_{w}$, for all the investigated samples. We find an increase of $a$ and a decrease of $b$ with increasing $\mathrm{L}_{\mathrm{w}}$. The first behavior is in agreement with theoretical predictions in the literature ${ }^{3}$ if we take into account that for thin InGaAs/GaAs quantum wells, an increase of the well width results in an
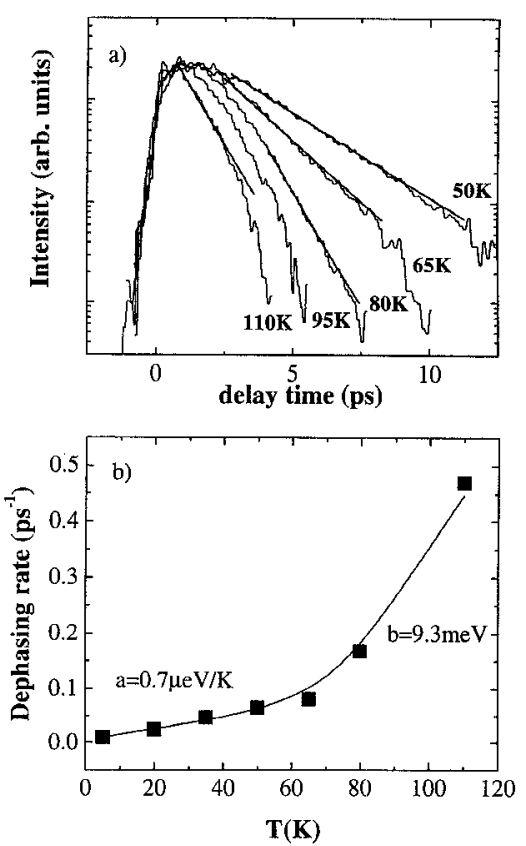

QWE3 Fig. 1. (a) Temperature dependence of TI-FWM at the heavy-hole exciton resonance in a $1.5-\mathrm{nm} \mathrm{In}_{0.18} \mathrm{Ga}_{0.82} \mathrm{As} / \mathrm{GaAs}$ single quantum well. Exponential fits of the decay are also indicated. (b) Dephasing rate as a function of the temperature in the sample of Fig. $1 \mathrm{a}$. The best fit of the data together with the deduced acoustic and optical phonon linewidth broadening coefficient (with linewidth in terms of half width at half maximum as in Ref. 1) are indicated.

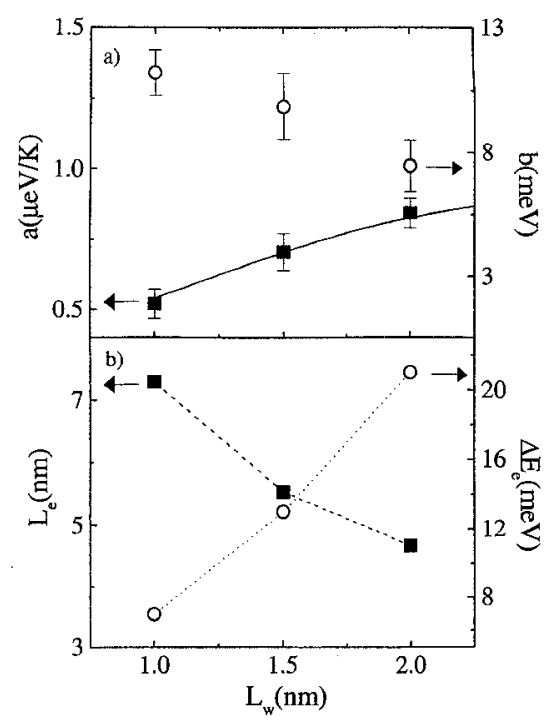

QWE3 Fig. 2. (a) Acoustic and optical phonon coefficients as functions of the well width for all samples. The solid line shows the well-width dependence of $1 / L_{e}$ (see Fig. 1b). (b) Full width at half maximum $L_{e}$ of the square of the electronic wave function and energy distance $\Delta \mathrm{E}_{\mathrm{e}}$ between the only electronic confined level in the well and the electron barrier potential as a function of the well width.

increased carrier confinement due to a re duced penetration into the GaAs barrier, as 
shown by the well-width dependence of exciton binding energies and linewidths. ${ }^{4}$

In Fig. $2 b$ the width $\mathrm{L}_{e}$ of the square of the electron wave function is reported as a function of $L_{w}$. The trend of $1 / L_{e}$ is shown in Fig. 1a as a solid line and results in very good agreement with the dependence of $a$ on $L_{w}$. In Fig. $2 \mathrm{~b}$ we also report the energy distance $\Delta \mathrm{E}_{\mathrm{e}}$ of the only confined electronic level in the quantum well from the barrier potential as a function of $\mathrm{L}_{\mathrm{w}}$. This trend suggests that the increasing of $b$ with decreasing $\mathrm{L}_{\mathrm{w}}$ is related to scattering of carriers via optical phonon absorption toward levels with increasing density of states in the barrier continuum.

*Fondazione Ugo Bordoni, via B. Castiglione 59, 00142 Roma, Italy

1. D. Gammon et al., Phys. Rev. B 51, 16785 (1995).

2. J. Erland et al., Phys. Rev. B 50, 15047 (1994).

3. H. Hillmer et al., Phys. Rev. B 39, 10901 (1989).

4. A. Patanè et al., Phys. Rev. B 52, 2784 (1995).

\section{QWE4}

$8: 45$ am

Ultrafast semiconductor differential transmission spectroscopy with broadband chirped puises

J. Kunde, U. Siegner, S. Arlt,

F. Morier-Genoud, U. Keller, Swiss Federal

Institute of Technology Zurich, Institute of Quantum Electronics, ETH Hönggerberg HPT, CH-8093 Zurich, Switzerland; E-mail: kunde@iqe.phys.ethz.ch

We demonstrate experimentally that chirping of broadband 15-fs pulses has drastic effects on the differential transmission (DT) response of bulk semiconductors for excitation well above the bandgap (see inset of Fig. 1). In particular, the rise and the initial fast decay of the spectrally integrated (SI) DT response and the shape of DT spectra are modified by chirp in an unexpected way. These new results have an impact on both fundamental studies and ultrafast switching applications as, e.g., mode locking of solid-state lasers. ${ }^{1}$

Experiments were performed on a 200-nm thick $\mathrm{Al}_{0.06} \mathrm{Ga}_{0.94} \mathrm{As}$ sample at $300 \mathrm{~K}$. Upchirping (low-energy components in the leading edge of the pulse) or down-chirping increases the pulse width from 15 fs to $60 \mathrm{fs}$.

The SI-DT in Fig. 1 shows a pronounced fast decay with a time constant of $20 \mathrm{fs}$ for down-chirped pulses, which is weaker for unchirped and absent for up-chirped pulses. The signal rises considerably faster for up-chirped than for down-chirped pulses even though the pulse widths are equal. This demonstrates that the rise of DT signals is not determined by the pulse width alone, as commonly assumed.

Spectrally resolved studies qualitatively $\mathrm{ex}$ plain these results. For unchirped pulses and negative time delay, the DT spectrum in Fig. 2 shows a spectral hole (bleaching, i.e., positive DT) ${ }^{2}$ redshifted from the maximum of the pump spectrum and induced absorption (negative DT) at the high-energy side of the pump. The same features were observed for

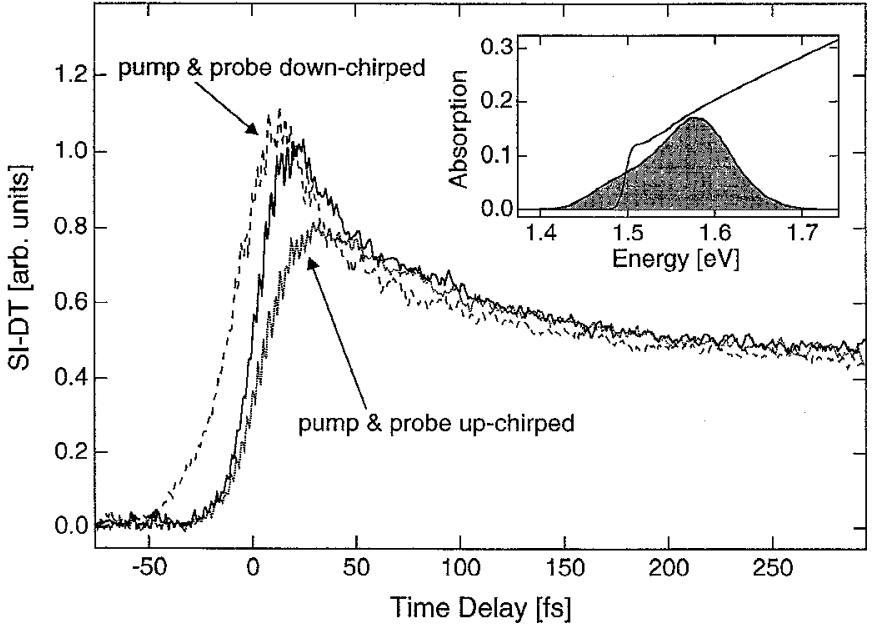

QWE4 Fig. 1. Spectrally integrated differential transmission for unchirped (solid), down-chirped (dashed), and up-chirped (dotted) pump and probe pulses; excitation density $\approx 3 \cdot 10^{17} \mathrm{~cm}^{-3}$. Pump and probe have identical spectra and are collinearly polarized. Inset: Absorption spectrum of the 200-nm thick $\mathrm{Al}_{0.06} \mathrm{Ga}_{0.94} \mathrm{As}$ sample at room temperature (solid line); pump spectrum (shaded).

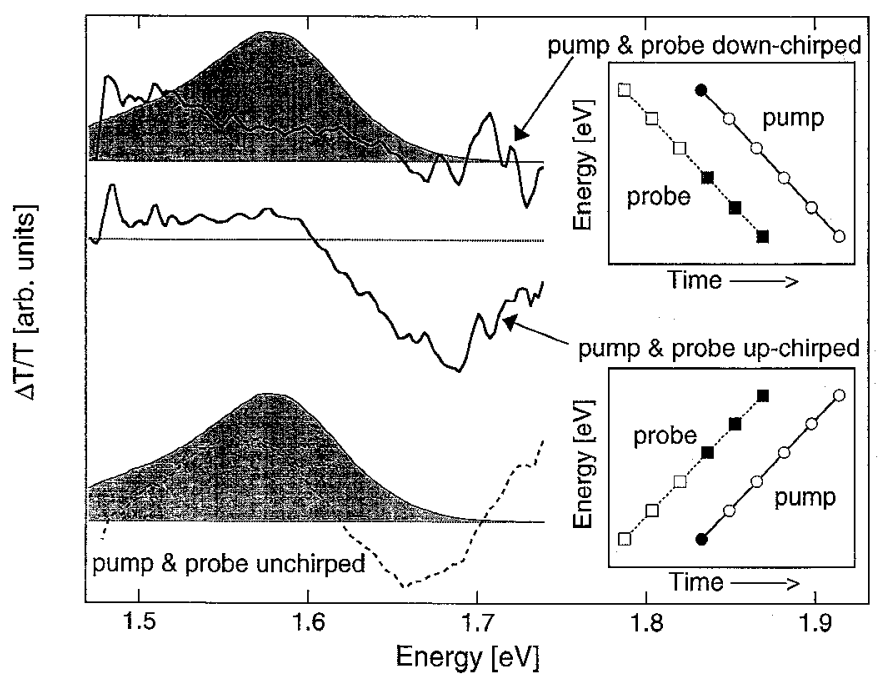

QWE4 Fig. 2. Differential transmission (DT) spectra for unchirped pump and probe pulses (dashed line) and for up- and down-chirped pump and probe pulses (solid lines) at negative time delay where the probe precedes the pump; excitation density $\approx 3 \cdot 10^{17} \mathrm{~cm}^{-3}$. The pump spectrum (shaded) is also shown. Insets: Schematic picture of the pulse frequency components versus time for up-and down-chirp. The transmission changes induced by the pump energy component marked by the filled circle are read out by the time-delayed probe energy components marked by the filled squares.

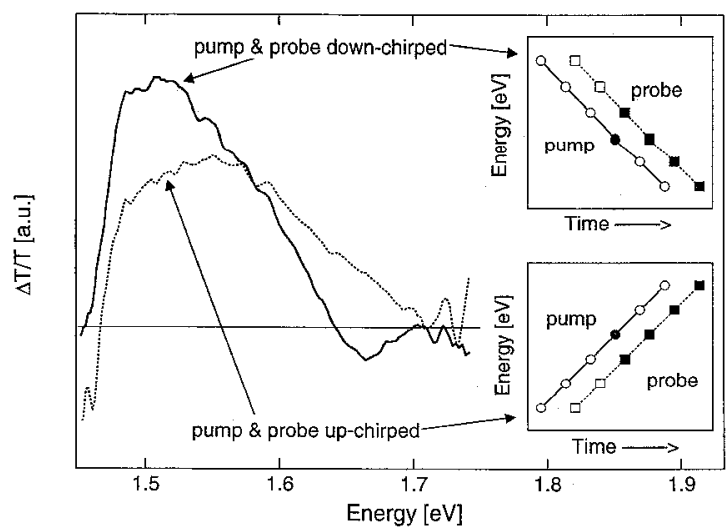

QWE4 Fig. 3. Differential transmission spectra for down-chirp (solid) and up-chirp (dotted) at the SI-DT maximum (small positive time delay); excitation density $\approx 3 \cdot 10^{17} \mathrm{~cm}^{-3}$. Insets: Schematic picture of the pulse frequency components versus time for up- and down-chirp. The transmission changes induced by the pump energy component marked by the filled circle are read out by the time-delayed probe energy components marked by the filled squares. 